$\operatorname{imsc} / 98 / 04 / 14$

\title{
Gauge field copies
}

\author{
Pushan Majumdar*, H.S.Sharatchandra $^{\dagger}$ \\ Institute of Mathematical Sciences, C.I.T campus Taramani. Madras 600-113
}

\begin{abstract}
The problem of $\mathrm{Wu}$-Yang ambiguities in 3 dimensions is related to the problem of existence of torsion free driebeins for an arbitrary potential. The ambiguity is only at the level of boundary conditions. We also find that in 3 dimensions, any smooth Yang-Mills field tensor can be uniquely written as the non-Abelian magnetic field of a smooth Yang-Mills potential.
\end{abstract}

PACS No(s). 11.15.-q 04.20.-q

*e-mail:pushan@imsc.ernet.in

${ }^{\dagger}$ e-mail:sharat@imsc.ernet.in 
Wu and Yang [1] gave an explicit example of two (gauge inequivalent) Yang-Mills potentials $\vec{A}_{i}(x)=\left\{A_{i}^{a}(x), a=1,2,3\right\}$ generating the same non-Abelian magnetic field

$$
\vec{B}_{i}[A](x)=\epsilon_{i j k}\left(\partial_{j} \vec{A}_{k}+\frac{1}{2} \vec{A}_{j} \times \vec{A}_{k}\right) .
$$

Since then there has been a wide discussion of the phenomenon in the literature [2 15]. We may refer to gauge potentials giving the same non-Abelian magnetic field, as gauge field copies in contrast to gauge equivalent potentials which generate magnetic fields related by a homogeneous gauge transformation. If we require all higher covariant derivatives of $B_{i}^{a}$ also match then there are effectively no gauge copies [11].

Deser and Wilczek [⿴囗十] first pointed out the consistency condition for $\vec{A}_{\mu}$ and $\vec{A}_{\mu}^{\prime}=\vec{A}_{\mu}+\vec{\Delta}_{\mu}$ to generate the same field strength. Using the Bianchi identity, they obtained that $\vec{\Delta}_{\mu}$ had to satisfy the equation

$$
\left[\tilde{\mathbf{F}}_{\mu \nu}, \Delta_{\nu}\right]=0
$$

where in 2 dimensions,

$$
\tilde{F}^{\mu \nu a b}=\frac{1}{2} \epsilon^{\mu \nu} \epsilon^{a b c} F_{\mu \nu}^{c}=M^{a b}
$$

and in 4 dimensions

$$
\tilde{F}^{\mu \nu a b}=\frac{1}{2} \epsilon^{\mu \nu \rho \sigma} \epsilon^{a b c} F_{\rho \sigma}^{c}=M^{a \mu, b \nu} .
$$

Treating this as an eigenvalue equation for $\Delta$, we have the condition for existence of nontrivial solutions of $\Delta$ is that the determinant of $\mathbf{M}$ is zero. In 2 dimensions the determinant corresponding to $\mathbf{M}$ vanishes identically and there $\Delta$ necessarily has non-trivial solutions. However in 4 dimensions this determinant in generically non-zero and there are hardly any gauge copies.

This sort of analysis exists only in even dimensions. In 3 Euclidean dimensions, we only get the constraint $\vec{B}_{i}[A] \times \vec{\Delta}_{i}=0$. This equation has many solutions, but this is only a consisitency condition. It does not mean that any $\vec{\Delta}_{i}$ satisfying this equation gives a gauge copy. Recently Freedman and Khuri [15] have exhibited several examples of continuous families of gauge field copies in $\mathrm{d}=3$. Their technique was to use a local map of the gauge field system into a spatial geometry with a second rank symmetric tensor $G_{i j}=B_{i}^{a} B_{j}^{a} \operatorname{det} B$ and a connection with torsion constructed from it.

We adopt a different method and directly ask the question as to how many different solutions (if any), does the system of equations defined by (1) have for any specified $\vec{B}_{i}(x)$. For that we proceed with the analysis using the Cauchy - Kowalevsky existence theorems on systems of partial differential equations. The equations for the gauge field copies are not a priori in the form where this theorem can be applied. However by reorganizing the equations a bit they can be brought to the form so that these theorems can be applied to that system. 


\section{EXISTENCE OF A FOR ARBITRARY B}

Let us first state the Cauchy - Kowalevsky existence theorem which we use [16]. Let a set of partial equations be given in the form

$$
\frac{\partial z_{i}}{\partial x_{1}}=\sum_{j=1}^{m} \sum_{r=2}^{n} G_{i j r} \frac{\partial z_{j}}{\partial x_{r}}+G_{i}
$$

for values $i=1, \ldots, m$, being $m$ equations in $m$ dependent variables ; the coefficients $G_{i j r}$ and the quantities $G_{i}$ are functions of all the variables, dependent and independent. Let $c_{1}, \ldots, c_{m}, a_{1}, \ldots, a_{n}$ be a set of values of $z_{1}, \ldots z_{m}, x_{1}, \ldots, x_{n}$ respectively, in the vicinity of which all the functions $G_{i j r}$ and $G_{i}$ are regular ; and let $\phi_{1}, \ldots, \phi_{m}$ be a be a set of functions of $x_{2}, \ldots, x_{n}$, which acquire values $c_{1}, \ldots, c_{m}$ respectively when $x_{2}=a_{2}, \ldots, x_{n}=a_{n}$, which are regular in the vicinity of these values of $x_{2}, \ldots, x_{n}$, and which are otherwise arbitrary. Then a system of integrals of the equations can be determined, which are regular functions of $x_{1}, \ldots, x_{n}$ in the vicinity of the values $x_{1}=a_{1}, x_{2}=a_{2}, \ldots, x_{n}=a_{n}$, and which acquire the values $\phi_{1}, \ldots, \phi_{m}$ when $x_{1}=a_{1}$; moreover, the system of integrals determined in accordance with these conditions, is the only system of integrals that can be determined as regular functions.

Our system of equations is

$$
\begin{aligned}
& \vec{B}_{1}=\partial_{2} \overrightarrow{A_{3}}-\partial_{3} \overrightarrow{A_{2}}+\overrightarrow{A_{2}} \times \overrightarrow{A_{3}} \\
& \vec{B}_{2}=\partial_{3} \overrightarrow{A_{1}}-\partial_{1} \overrightarrow{A_{3}}+\overrightarrow{A_{3}} \times \overrightarrow{A_{1}} \\
& \vec{B}_{3}=\partial_{1} \overrightarrow{A_{2}}-\partial_{2} \overrightarrow{A_{1}}+\overrightarrow{A_{1}} \times \overrightarrow{A_{2}}
\end{aligned}
$$

where $\vec{B}_{1}, \vec{B}_{2}$ and $\vec{B}_{3}$ are treated as given variables and we want to solve for $\overrightarrow{A_{1}}, \overrightarrow{A_{2}}$ and $\vec{A}_{3}$. With this definition of the $B^{\prime} s$, the bianchi identity $D_{i} B_{i}=0$ follows automatically. However the existence theorem cannot be applied directly to this set of equations. For that we rewrite the equations in a different way. Consider

$$
\begin{aligned}
& \partial_{3} \overrightarrow{A_{2}}=\partial_{2} \overrightarrow{A_{3}}+\overrightarrow{A_{2}} \times \overrightarrow{A_{3}}-\overrightarrow{B_{1}} \\
& \partial_{3} \overrightarrow{A_{1}}=\partial_{1} \overrightarrow{A_{3}}-\overrightarrow{A_{3}} \times \overrightarrow{A_{1}}+\overrightarrow{B_{2}} .
\end{aligned}
$$

The existence theorem implies that we have solution for $\overrightarrow{A_{1}}$ and $\overrightarrow{A_{2}}$ for any specified $\overrightarrow{B_{1}}, \overrightarrow{B_{2}}$ and $\overrightarrow{A_{3}}$. But $\overrightarrow{A_{1}}$ and $\overrightarrow{A_{2}}$ so obtained have to satisfy equation (8). Is this always possible with some choice of $\overrightarrow{A_{3}}$, and if yes, is the choice of $\overrightarrow{A_{3}}$ unique? To address this question, we presume that the initial data on $x_{3}=0$ satisfies equations (6)-(8). This is always possible for any given $\vec{B}_{i}(x)$ as follows from the analysis of the 1+1-dimensional case. Then equation (8) may be equivalently replaced by another equation obtained by applying $\partial_{3}$ on it and using (6) $-(\sqrt{7})$. This is just the Bianchi identity. We write it in the form

$$
\vec{A}_{3} \times \vec{B}_{3}=-\partial_{3} \vec{B}_{3}-\partial_{2} \vec{B}_{2}-\vec{A}_{2} \times \vec{B}_{2}-\partial_{1} \vec{B}_{1}-\vec{A}_{1} \times \vec{B}_{1}
$$

Now let us decompose $\overrightarrow{A_{3}}$ in directions parallel and perpendicular to $\vec{B}_{3}$,

$$
\overrightarrow{A_{3}}=\alpha \overrightarrow{B_{3}}+\overrightarrow{A_{3 \perp}} .
$$


In the generic case, where $|\vec{B}| \neq 0$, equation (11) determines $\vec{A}_{3 \perp}$ entirely. Taking the cross product of (11) with $\vec{B}_{3}$, we get,

$$
\overrightarrow{A_{3}}=\alpha \vec{B}_{3}-\frac{1}{\left|\vec{B}_{3}\right|^{2}} \vec{B}_{3} \times\left[\left(\vec{A}_{2} \times \vec{B}_{2}\right)+\left(\vec{A}_{1} \times \vec{B}_{1}\right)+\left(\partial_{i} \vec{B}_{i}\right)\right] .
$$

where $\alpha$ can be arbitrarily chosen.

We now address the question whether $\alpha$ can also be determined. Taking the dot product of (11) with $\vec{B}_{3}$, we get,

$$
\vec{B}_{3} \cdot \partial_{i} \vec{B}_{i}+\left(\vec{B}_{3} \times \vec{B}_{1}\right) \cdot \vec{A}_{1}+\left(\vec{B}_{3} \times \vec{B}_{2}\right) \cdot \vec{A}_{2}=0 .
$$

This is a constraint which $\overrightarrow{A_{1}}$ and $\overrightarrow{A_{2}}$ have to satisfy. It is satisfied on $x_{3}=0$. In order that it is satisfied at all $x_{3}$, we apply $\partial_{3}$ on (14) and use (9) and (10). We obtain

$$
\begin{gathered}
-\left(\partial_{1} \vec{A}_{3}-\vec{A}_{3} \times \vec{A}_{1}+\vec{B}_{2}\right) \cdot\left(\vec{B}_{1} \times \vec{B}_{3}\right)-\vec{A}_{1} \cdot \partial_{3}\left(\vec{B}_{1} \times \vec{B}_{3}\right) \\
-\left(\partial_{2} \vec{A}_{3}+\vec{A}_{2} \times \vec{A}_{3}-\vec{B}_{1}\right) \cdot\left(\vec{B}_{2} \times \vec{B}_{3}\right)-\vec{A}_{2} \cdot \partial_{3}\left(\vec{B}_{2} \times \vec{B}_{3}\right) \\
+\partial_{3}\left(\partial_{i} \vec{B}_{i}\right) \cdot \vec{B}_{3}+\left(\partial_{i} \vec{B}_{i}\right) \cdot\left(\partial_{3} \vec{B}_{3}\right)=0
\end{gathered}
$$

Now we can substitute the expression for $\vec{A}_{3}$ from (13). Note that in this substitution, the derivatives do not act on $\alpha$ since in that case we get terms $\vec{B}_{3} \cdot \vec{B}_{1} \times \vec{B}_{3}$ and $\vec{B}_{3} \cdot \vec{B}_{2} \times \vec{B}_{3}$ which vanish. We get the coefficient of $\alpha$ as $\left(D_{1}[A] \vec{B}_{3}\right) \cdot\left(\vec{B}_{1} \times \vec{B}_{3}\right)+\left(D_{2}[A] \vec{B}_{3}\right) \cdot\left(\vec{B}_{2} \times \vec{B}_{3}\right)$. Whenever this coefficient is non-zero, the linear equation for $\alpha$ is invertible and this explicitly gives us $\alpha$ as a function of $\overrightarrow{A_{1}}, \overrightarrow{A_{2}}$ and $\vec{B}_{i}$. Generically we do not expect any problem in solving for $\alpha$.

We now have $\vec{A}_{3}$ as a local function of $\overrightarrow{A_{1}}, \overrightarrow{A_{2}}$ and $\vec{B}_{i}$ 's and we can substitute for it in (9-10). We further expect that the field configurations are mostly non-vanishing so that the coefficients $G_{i j r}$ and $G_{i}$ are regular and we can apply the theorem to get $\overrightarrow{A_{1}}, \overrightarrow{A_{2}}$ and hence $\overrightarrow{A_{3}}$ as unique functionals of $\vec{B}_{i}(x)$.

Alternatively we could consider the system of equations

$$
\begin{aligned}
\partial_{3} \overrightarrow{A_{2}}= & \partial_{2} \overrightarrow{A_{3}}+\overrightarrow{A_{2}} \times \overrightarrow{A_{3}}-\overrightarrow{B_{1}} \\
\partial_{3} \overrightarrow{A_{1}}= & \partial_{1} \overrightarrow{A_{3}}-\overrightarrow{A_{3}} \times \overrightarrow{A_{1}}+\overrightarrow{B_{2}} \\
\partial_{3}\left(\overrightarrow{A_{3}} \times \overrightarrow{B_{3}}\right)= & -\left(\partial_{1} \overrightarrow{A_{3}}-\overrightarrow{A_{3}} \times \overrightarrow{A_{1}}+\overrightarrow{B_{2}}\right) \times \vec{B}_{1} \\
& -\left(\partial_{2} \vec{A}_{3}+\overrightarrow{A_{2}} \times \overrightarrow{A_{3}}-\overrightarrow{B_{1}}\right) \times \vec{B}_{2} \\
& -\vec{A}_{1} \times \partial_{3} \vec{B}_{1}-\partial_{3}\left(\partial_{i} \vec{B}_{i}\right)-\vec{A}_{2} \times \partial_{3} \vec{B}_{2} \\
\partial_{3}\left(\overrightarrow{A_{3}} \cdot \vec{B}_{3}\right)= & \partial_{3}\left(\left|\vec{B}_{3}\right|^{2} \alpha\left(\overrightarrow{A_{1}}, \vec{A}_{2}, \vec{B}_{i}\right)\right) .
\end{aligned}
$$

Here in the last equation $\alpha\left(\vec{A}_{1}, \vec{A}_{2}, \vec{B}_{i}\right)$ is to be replaced by the expression obtained for $\alpha$ from equation (15) and $\partial_{3} \overrightarrow{A_{1}}$ and $\partial_{3} \overrightarrow{A_{2}}$ are to be replaced using (16) and (17). This system of equations is in the form where the Cauchy-Kowalevsky theorem can be applied and this system uniquely determines all the unknown variables once the initial data is specified. The first two equations contain the six unknowns $\overrightarrow{A_{1}}$ and $\overrightarrow{A_{2}}$. The third one contains the two components of $\vec{A}_{3}$ transverse to $\vec{B}_{3}$ and the fourth one has the component of $\vec{A}_{3}$ parallel to $\vec{B}_{3}$. Thus all the nine degrees of freedom are uniquely determined. Therefore generically there are no gauge field copies. The only ambiguity in the choice of the potential is limited to a subspace which specifies the initial conditions as required in the theorem. 


\section{EXISTENCE OF TORSION FREE DRIEBEINS FOR ARBITRARY A}

In this section we address the question whether there exists any continuous family of potentials which generate the same magnetic field. Let $\vec{A}_{i}$ and $\vec{A}_{i}+\epsilon \vec{e}_{i}$ generate the same magnetic field, where $\epsilon$ is a small parameter. Then $\vec{e}_{i}$ satisfies the equation

$$
\epsilon_{i j k}\left(\partial_{j} \vec{e}_{k}+\vec{A}_{j} \times \vec{e}_{k}\right)=0 .
$$

This is precisely the equation for a driebein $\vec{e}_{i}$ to have zero torsion with respect to the connection one form $\vec{A}_{i}$. Thus we are asking if there exists a driebein with zero torsion for a given arbitrary connection one form. This is an important question in the context of general relativity. We also have a consistency condition by taking the covariant derivative of this equation. That is given by

$$
\vec{B}_{k} \times \vec{e}_{k}=0
$$

Let us rewrite the equations in a more convenient way. We take our system of equations as

$$
\begin{aligned}
& \partial_{3} \vec{e}_{2}=\partial_{2} \vec{e}_{3}+\vec{A}_{2} \times \vec{e}_{3}-\overrightarrow{A_{3}} \times \overrightarrow{e_{2}} \\
& \partial_{3} \vec{e}_{1}=\partial_{1} \vec{e}_{3}+\vec{A}_{1} \times \vec{e}_{3}-\overrightarrow{A_{3}} \times \overrightarrow{e_{1}}
\end{aligned}
$$

and the consistency condition (21). This set is equivalent to the set of equations (20). As in the previous case, we first look at the consistency condition. Let us decompose $\vec{e}_{3}$ as

$$
\vec{e}_{3}=\beta \vec{B}_{3}+\vec{e}_{3 \perp}
$$

Again (21) fixes for us $\vec{e}_{3 \perp}$ in terms of the magnetic fields (in the generic case $\vec{B}_{3} \neq 0$ ). We get,

$$
\vec{e}_{3}=\beta \vec{B}_{3}-\frac{1}{\left|\vec{B}_{3}\right|} \vec{B}_{I} \times \vec{e}_{I}
$$

where $I$ goes over 1,2 . Now we can substitute this form of $\vec{e}_{3}$ in the equations (22-23). We obtain $\vec{e}_{1}$ and $\vec{e}_{2}$ as unique functions of $\beta$ and the magnetic fields. However this $\vec{e}_{1}$ and $\vec{e}_{2}$ have to satisfy the consistency conditions

$$
\vec{B}_{3} \cdot \vec{B}_{I} \times \vec{e}_{I}=0
$$

where again $I$ goes over 1,2. Taking $\partial_{3}$ of equation (26), we get, using (22) and (23)

$$
D_{3}\left(\vec{B}_{3} \times \vec{B}_{I}\right) \cdot \vec{e}_{I}+\vec{B}_{I} \cdot \vec{B}_{3} \times D_{I} \vec{e}_{3}=0
$$

Putting in the expression of $\vec{e}_{3}$, we get a linear equation for $\beta$

$$
D_{3}\left(\vec{B}_{3} \times \vec{B}_{I}\right) \cdot \vec{e}_{I}+\left(\vec{B}_{I} \times \vec{B}_{3}\right) \cdot\left(D_{I} \vec{B}_{3}\right) \beta-\left(\vec{B}_{I} \times \vec{B}_{3}\right) \cdot D_{I}\left[\frac{1}{\left|\vec{B}_{3}\right|}\left(\vec{B}_{J} \times \vec{e}_{J}\right)\right]=0
$$

This equation can be inverted to solve for $\beta$ as a function of $\vec{e}_{1}, \vec{e}_{2}, \overrightarrow{A_{1}}, \overrightarrow{A_{2}}$ and $\vec{B}_{i}$ whenever $\left(\vec{B}_{I} \times \vec{B}_{3}\right) \cdot\left(D_{I} \vec{B}_{3}\right)$ is non-zero. 
Formally we could have also looked at the set of equations

$$
\begin{aligned}
\partial_{3} \vec{e}_{2}= & \partial_{2} \vec{e}_{3}+\vec{A}_{2} \times \vec{e}_{3}-\vec{A}_{3} \times \vec{e}_{2} \\
\partial_{3} \vec{e}_{1}= & \partial_{1} \vec{e}_{3}+\vec{A}_{1} \times \vec{e}_{3}-\vec{A}_{3} \times \vec{e}_{1} \\
\partial_{3}\left(\vec{B}_{3} \times \vec{e}_{3}\right)= & -\left(\partial_{3} \vec{B}_{2}\right) \times \vec{e}_{2}-\left(\partial_{3} \vec{B}_{1}\right) \times \vec{e}_{1} \\
& -\vec{B}_{2} \times\left(\partial_{2} \vec{e}_{3}+\vec{A}_{2} \times \vec{e}_{3}-\vec{A}_{3} \times \vec{e}_{2}\right) \\
& -\vec{B}_{1} \times\left(\partial_{1} \vec{e}_{3}+\vec{A}_{1} \times \vec{e}_{3}-\vec{A}_{3} \times \vec{e}_{1}\right) \\
\partial_{3}\left(\vec{B}_{3} \cdot \vec{e}_{3}\right)= & \partial_{3}\left(\left|\vec{B}_{3}\right|^{2}\right) \beta\left(\vec{e}_{1}, \vec{e}_{2}, \vec{A}_{1}, \vec{A}_{2}, \vec{B}_{i}\right) .
\end{aligned}
$$

In the last equation, $\beta$ has to be replaced by its solution from (28) and $\partial_{3} e_{I}$ is to be substituted from (29) and (30).

We expect the non-Abelian potentials and magnetic fields are smooth and non-vanishing so that the coeffiecient functions for the set of differential equations are regular. Applying the Cauchy-Kowalevsky theorem to this set, we get a unique smooth solution for $\vec{e}_{1}, \vec{e}_{2}$ and $\vec{e}_{3}$. Thus for any potential there is a torsion free driebein, and the only ambiguity is in the choice of the driebein to fix the initial conditions required by the theorem.

\section{AN EXPLICIT CALCULATION}

We now illustrate these results by an explicit calculation for the special case $A_{i}^{a}=\delta_{i}^{a}$. In momentum space, the equation looks like

$$
\epsilon_{i j k}\left(-i p_{j} \delta^{a c}+\epsilon_{a b c} \delta_{j}^{b}\right) e_{k}^{c}(p)=0
$$

or

$$
\left(-i \epsilon_{i j k} p_{j} \delta^{a c}+\delta_{i}^{a} \delta_{k}^{c}-\delta_{i}^{c} \delta_{k}^{a}\right) e_{k}^{c}(p)=0
$$

In three dimensions we can choose three orthogonal vectors. We choose three such vectors as $(\vec{p}, \vec{n}, \vec{m})$ where $\vec{p}$ coincides with the $\vec{p}$ which appears in the equation and $\vec{n}$ and $\vec{m}$ are unit vectors. We also orient $(\vec{p}, \vec{n}, \vec{m})$ such that $\vec{p} \times \vec{m}=|\vec{p}| \vec{n}$ and $\vec{p} \times \vec{n}=-|\vec{p}| \vec{m}$. Next we write a general solution for $e_{k}^{c}$ in terms of the dyad basis as

$$
\begin{gathered}
e_{k c}=a_{1} n_{c} m_{k}+a_{2} n_{k} m_{c}+a_{3} n_{k} n_{c}+a_{4} m_{k} m_{c} \\
+a_{5} p_{c} m_{k}+a_{6} p_{k} m_{c}+a_{7} p_{c} n_{k}+a_{8} p_{k} n_{c}+a_{9} p_{k} p_{c},
\end{gathered}
$$

where $a_{i}$ 's are unknown coefficients to be determined.

Substituting the solution in the equation, we get various relations among the coefficients. $a_{5}, a_{6}, a_{7}, a_{8}$ and $a_{9}$ turn out to be zero identically. In addition we get

$$
-i|\vec{p}| a_{1}=-i|\vec{p}|^{3} a_{2}=a_{3}=|\vec{p}|^{2} a_{4} .
$$

Therefore, we get a non-zero solution only if

$$
|\vec{p}|=1,
$$


in which case,

$$
-i a_{1}=-i a_{2}=a_{3}=a_{4}=a
$$

Thus the general solution is

$$
e_{i b}(x)=\int d \Omega a(\Omega) e^{i \hat{p} \cdot x}(\hat{m}+i \hat{n})_{i}(\hat{m}-i \hat{n})_{b}
$$

Here the integration is over all directions of the vector $\hat{p}$. The solutions have an arbitrary function $a(\Omega)$. We may fix $a(\Omega)$ by using initial data on $x_{3}=0$ surface. This may be interpreted as the arbitrary choice of $\vec{e}_{i}(x)$ at the boundary. However if we require $\vec{e}_{i}(x)$ vanishes rapidly at infinity, there may not be any solutions. Thus gauge copies would be absent in this case.

A similar exercise can be carried out for any constant vector potential and gives an identical result.

\section{CONCLUSIONS}

In this chapter we have looked at two problems regarding the existence of non-Abelian vector potentials. First we asked the question if there exists a vector potential for any arbitrary magnetic field. We found that there are many choices of $\vec{A}_{i}(x)$ on the $x_{3}=0$ surface which reproduces $\vec{B}_{i}(x)$ on the surface. (This is the gauge field ambiguity in $1+1$ dimensions.) For each such boundary condition on $\vec{A}_{i}(x)$ we have seen (in the generic case) that there is a unique potential $\vec{A}_{i}(x)$ which reproduces the given magnetic field everywhere. The non-Abelian Bianchi identity does not constrain the non-Abelian magnetic fields in contrast to the abelian case. The ambiguity in the choice of the potentials is (in the generic case) only due to the ambiguity in $\vec{A}_{i}(x)$ on the $x_{3}=0$ surface. Thus it is related to the gauge copy problem in $1+1$ dimensions.

We thank Professors Ramesh Anishetty and K. Mariwalla for helpful discussions and Prof. P.P.Divakaran for a useful comment. 


\section{REFERENCES}

[1] T.T. Wu and C.N. Yang, Phys. Rev. D12, 3845 (1975).

[2] C.H. Gu and C.N. Yang, Sci. Sin. 18, 484 (1975); Sci. Sin. 20, 47 (1977).

[3] C.L. Shen, Fudan Journal (Natural Science) 2, 61 (1976).

[4] S. Deser and F.Wilczek, Phys. Lett. 65B, 391 (1976).

[5] R. Roskies, Phys. Rev. D15, 1731 (1977).

[6] M. Calvo, Phys. Rev. D15, 1733 (1977).

[7] M.B. Halpern, Phys. Rev. D16, 1798 (1977); Nucl. Phys. B139, 477 (1978).

[8] S. Coleman, Phys. Lett. 70B, 59 (1977).

[9] S. Solomon, Nucl. Phys. B147, 174 (1979).

[10] C.G. Bollini, J.J.Giambiagi and J.Tiomno, Phys. Lett. 83B, 185 (1979).

[11] L.S. Brown and W.I. Weisberger, Nucl. Phys. B157, 285 (1979).

[12] S.Deser and W. Drechsler, Phys. Lett. 86B, 189 (1979).

[13] M. Mostow, Commun. Math. Phys. 78, 137 (1979).

[14] F.A.Doria, Commun. Math. Phys. 79, 435 (1981); J. Math. Phys. 22, 2943 (1981).

[15] D.Z.Freedman and R.R.Khuri, Phys. Lett. B329, 263 (1994).

[16] A.R.Forsyth, "Theory of differential equations" Part IV, Dover Publications Inc. 1959, pp. 11 